\title{
Multicellularity Drives the Evolution of Sexual Traits
}

\author{
Erik R. Hanschen, ${ }^{1,2, \star}$ Matthew D. Herron, ${ }^{3}$ John J. Wiens, ${ }^{1}$ Hisayoshi Nozaki, \\ and Richard E. Michod ${ }^{1}$
}

1. Department of Ecology and Evolutionary Biology, University of Arizona, Tucson, Arizona 85721; 2. Division of Bioscience, Los Alamos National Laboratory, Los Alamos, New Mexico 87545; 3. School of Biological Sciences, Georgia Institute of Technology, Atlanta, Georgia 30332; 4. Department of Biological Sciences, University of Tokyo, Tokyo 113-0033, Japan

Submitted September 5, 2017; Accepted February 28, 2018; Electronically published July 10, 2018

Online enhancements: appendix. Dryad data: http://dx.doi.org/10.5061/dryad.rr3667m.

\begin{abstract}
From the male peacock's tail plumage to the floral displays of flowering plants, traits related to sexual reproduction are often complex and exaggerated. Why has sexual reproduction become so complicated? Why have such exaggerated sexual traits evolved? Early work posited a connection between multicellularity and sexual traits such as anisogamy (i.e., the evolution of small sperm and large eggs). Anisogamy then drives the evolution of other forms of sexual dimorphism. Yet the relationship between multicellularity and the evolution of sexual traits has not been empirically tested. Given their extensive variation in both multicellular complexity and sexual systems, the volvocine green algae offer a tractable system for understanding the interrelationship of multicellular complexity and sex. Here we show that species with greater multicellular complexity have a significantly larger number of derived sexual traits, including anisogamy, internal fertilization, and secondary sexual dimorphism. Our results demonstrate that anisogamy repeatedly evolved from isogamous multicellular ancestors and that anisogamous species are larger and produce larger zygotes than isogamous species. In the volvocine algae, the evolution of multicellularity likely drives the evolution of anisogamy, and anisogamy subsequently drives secondary sexual dimorphism. Multicellularity may set the stage for the overall diversity of sexual complexity throughout the Tree of Life.
\end{abstract}

Keywords: sex, volvocine green algae, multicellularity, ancestral state reconstruction, anisogamy, sexual dimorphism.

\section{Introduction}

The diversity of sexual reproduction throughout the Tree of Life is astonishing - from the fusion of monomorphic gametes in single-celled organisms to the bizarre mating rituals and strikingly dimorphic plumage of the birds of paradise. What explains this diversity of sexual biology and the evolution of

\footnotetext{
* Corresponding author; email: hanschen@lanl.gov.
}

ORCIDs: Hanschen, http://orcid.org/0000-0002-6595-4564; Herron, http:// orcid.org/0000-0002-9578-0972; Nozaki, http://orcid.org/0000-0002-9308-4498; Michod, http://orcid.org/0000-0002-7782-0379.

Am. Nat. 2018. Vol. 192, pp. E93-E105. (C) 2018 by The University of Chicago. 0003-0147/2018/19203-57935\$15.00. All rights reserved.

DOI: $10.1086 / 698301$ sexual traits? More elaborate forms of sexual reproduction depend on female and male sexes, which arise after the evolution of anisogamy (Lehtonen et al. 2016b). Anisogamy, the evolution of unequal-sized male and female gametes (numerous, smaller sperm and fewer, larger eggs), has almost certainly evolved repeatedly from isogamous ancestors, those with equal-sized gametes (Maynard Smith 1982; Togashi and Cox 2011; Beukeboom and Perrin 2014). Because males do not produce offspring, sex in anisogamous species has a twofold cost, also known as the cost of males, compared with sex in isogamous relatives (Maynard Smith 1978). Despite this cost, most multicellular taxa are anisogamous (Bell 1982), thus raising the question, Why are multicellular taxa mostly anisogamous?

The anisogamy gateway hypothesis attempts to explain the prevalence of anisogamy despite its costs by proposing that anisogamy acts as a "gateway" for the diversity of sexual forms we observe today (Lehtonen et al. 2016a). This hypothesis has three major components. First, the evolution of anisogamy may be favored by disruptive selection for increased number of gametes and increased survival of zygotes (Parker et al. 1972; Bell 1978, 1982), where zygote survival is an increasing function of zygote size. Multicellular species are hypothesized to require increased zygotic provisioning to provide nourishment for a larger embryo (Parker et al. 1972; Bulmer and Parker 2002), selecting for increased zygote size. As zygote size increases, disruptive selection for increased number of gametes and increased zygote survival disrupts the isogamous evolutionary stable state, and anisogamy evolves. Anisogamy subsequently sets the stage for the secondary evolution of sex roles and sexual dimorphism (Bateman 1948; Parker et al. 1972; Lehtonen et al. 2016b). Second, once anisogamy has evolved, males producing fewer, larger, isogametelike sperm are outcompeted by males producing numerous, smaller sperm. Therefore, sperm competition irreversibly maintains anisogamy (Parker 1982). Third and last, invasion of an isogamous or anisogamous sexual population of obligately asexual individuals is prevented by previously evolved con- 
straints (Lehtonen et al. 2016a). Through these three components, this hypothesis attempts to explain how multicellularity causes the irreversible evolution of anisogamy, which subsequently causes the evolution of sexual dimorphism.

While the anisogamy gateway hypothesis predicts that multicellularity causes anisogamy, an alternative hypothesis postulates the opposite relationship. In this view, anisogamy evolves in unicellular ancestors and subsequently drives the evolution of multicellularity and germ-soma cellular differentiation (Woodland 2016). The evolution of anisogamy results in larger, heavier eggs. These larger eggs require additional provisions, which may be provided by the origin of numerous somatic cells. Thus, anisogamy is thought to subsequently cause the simultaneous evolution of multicellularity and increased complexity (germ-soma cellular differentiation), to support increasing egg size. This is predicted to lead to a positive correlation between anisogamy and germ-soma cellular differentiation, with anisogamy evolving before germ-soma cellular differentiation (Woodland 2016).

The volvocine algae are a uniquely valuable model system for studying the joint evolution of sex and multicellularity. Previous studies have shown that the ancestor of the group was unicellular (Herron and Michod 2008), and multicellularity evolved relatively recently at $\sim 230$ million years ago (Herron et al. 2009). Furthermore, several volvocine genomes have been sequenced (Merchant et al. 2007; Prochnik et al. 2010; Hanschen et al. 2016; Featherston et al. 2018). All species are haploid and facultatively sexual (fig. A1; figs. A1, A2 are available online). Finally, the extant species exhibit remarkable morphological diversity, spanning unicellular species, multicellular species with undifferentiated cells, and multicellular species with differentiated cells (fig. $1 A-1 F$ ).

Sexual morphology in the volvocine algae includes isogamy (equal-sized gametes produced by self-incompatible $[-]$ and $[+]$ mating types), anisogamy (smaller sperm, bundled into multicellular "sperm packets," produced by males and larger, flagellated, unicellular eggs produced by females), and oogamy (a form of anisogamy where eggs are unflagellated and much larger than sperm; figs. $1 \mathrm{H}, \mathrm{A} 1)$. Fusion and fertilization of these gametes can occur both externally and internally to a female colony (figs. 1I, A1). External fertilization requires gametes finding and fertilizing each other (fig. 1I). Internal fertilization requires a multicellular sperm packet to find and penetrate the multicellular female colony wall and dissociate into individual sperm. Individual sperm must then swim through the extracellular matrix to find and fertilize a unicellular egg (fig. 1I). As fertilization that is internal to a multicellular body is possible only after the evolution of multicellularity, we predict internal fertilization to be positively correlated with the evolution of multicellular complexity.

In the volvocine algae, a diploid zygospore that is resistant to heat, cold (winter), and desiccation is formed after fertilization (fig. A1; Kirk and Kirk 1986; Kirk 1998). In some species, four meiotic progeny enter the asexual life cycle on germination. In other species, a reduced number of meiotic progeny (one or rarely two) enter the asexual life cycle, while the remaining meiotic products are discarded as polar bodies (figs. 1G, A1). A reduced number of meiotic products may be related to the evolution of multicellularity because it increases zygotic provisioning per sexual offspring, similar to the effect of anisogamy. When a reduced number of surviving meiotic products evolves, zygotic provisioning per sexual offspring may increase substantially, assuming the zygote size remains constant or increases.

Secondary sexual dimorphism is exhibited through extrafertile females, multicellular sperm packets, and dwarf males. Extrafertile females have a number of eggs in a female colony that is at least more than twice the number of reproductive gonidia in an asexual colony (sometimes called "special females"; Smith 1944; Nozaki and Itoh 1994; Nozaki 2003; figs. 1J, A1). Dwarf males are male colonies that are one-tenth to one-half the size of female colonies (Coleman 2012; figs. $1 \mathrm{~K}$, A1). As multicellularity is hypothesized to drive the evolution of anisogamy (Parker et al. 1972; Bulmer and Parker 2002) and anisogamy drives female and male sex roles (Lehtonen et al. 2016b), we expect sexual dimorphism and multicellular complexity to be positively correlated in the volvocine algae.

The volvocine algae have been used previously to test theories on the evolution of anisogamy. Knowlton (1974) and Bell (1978) observed that larger species tended to be anisogamous. Bell (1985) provided evidence that egg size and gamete dimorphism are positively correlated with increasing body size; however, this analysis was not corrected for phylogenetic nonindependence. Later, Randerson and Hurst (2001) analyzed the correlations between body size and egg size and between body size and gamete dimorphism in a phylogenetic context. However, their results were highly sensitive to the phylogeny and statistical methods used (Randerson and Hurst 2001). These authors used poorly supported phylogenies that are incongruent with recent phylogenies based on additional data and estimated with more advanced methods (Herron and Michod 2008). Therefore, the relationships among these traits remain uncertain.

To investigate how the evolution of multicellular complexity affects the evolution of sexual traits, we used ancestral state reconstruction to infer the evolutionary histories of six sexual traits in the volvocine algae: (1) a reduced number of meiotic products, (2) internal fertilization, (3) anisogamy, (4) oogamy, (5) extrafertile females, and (6) dwarf males. We utilize reconstructed evolutionary histories of volvocine green algae to test the following predictions: (1) anisogamy and other sexual traits evolved in a multicellular ancestor (Bulmer and Parker 2002), (2) sexual traits (including a reduced number of meiotic products hatching, internal fertilization, anisogamy, and sexual dimorphism) positively correlate with multicellular 

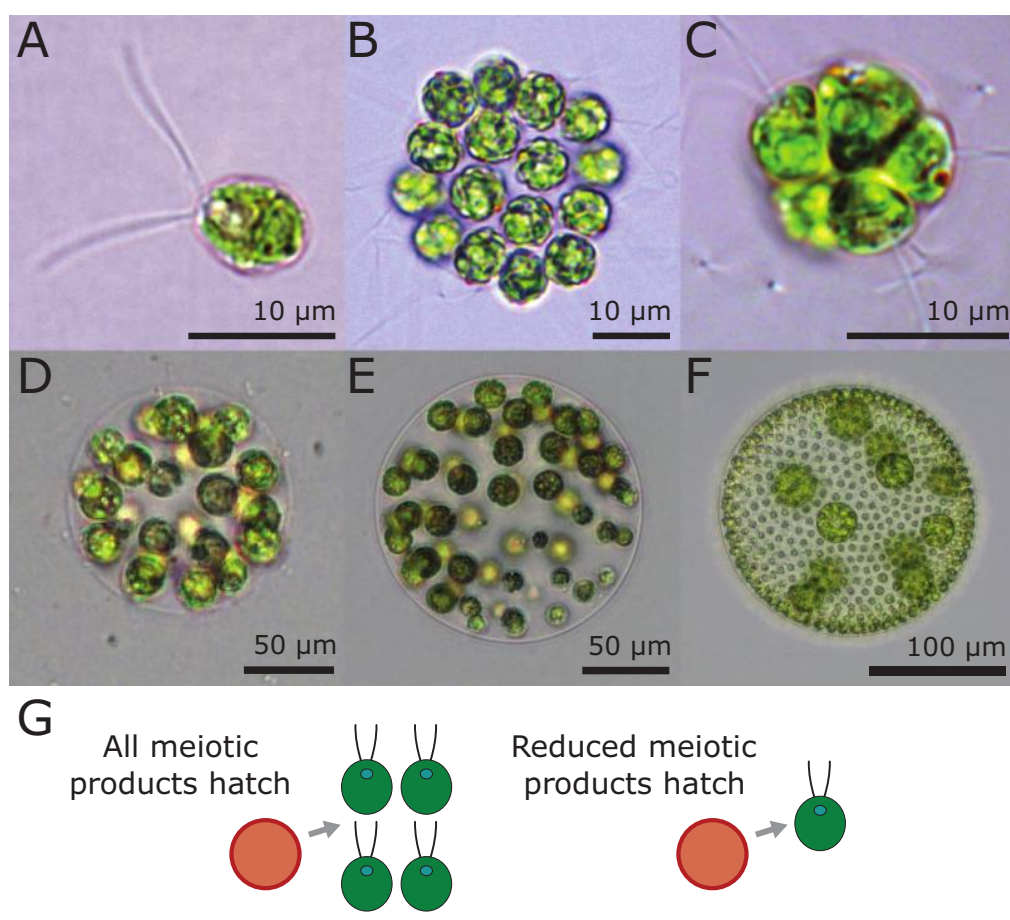

Reduced meiotic products hatch

$\mathrm{H}$

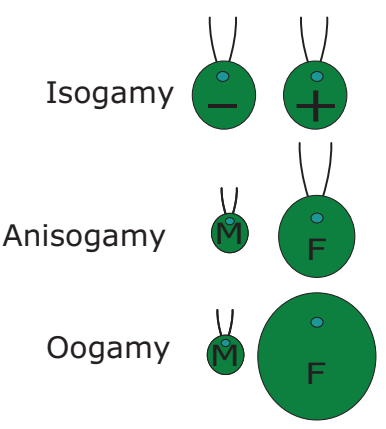

I

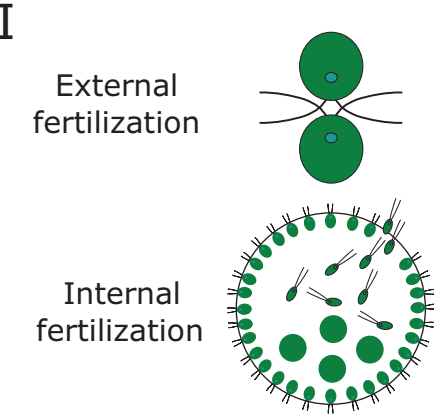

J

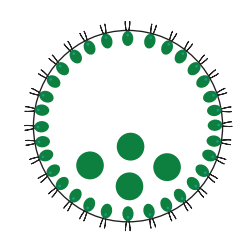

K

Female

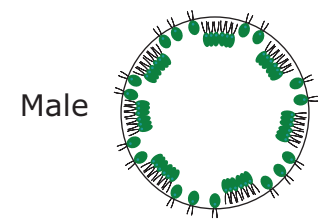

Extra-

fertile female

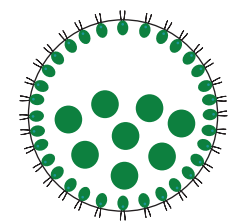

Dwarf

male

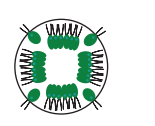

Figure 1: Exemplar species and diversity of sexual systems in the volvocine algae. A, Unicellular Chlamydomonas reinhardtii. B, Undifferentiated Gonium pectorale Russia. C, Undifferentiated Pandorina morum. D, Undifferentiated Eudorina elegans. E, Soma-differentiated Pleodorina californica. F, Germ- and soma-differentiated Volvox carteri f. nagariensis. G, A desiccation-resistant diploid zygospore produces four meiotic products (left) or a reduced number of meiotic products (right). H, Isogamy (equal-sized gametes), anisogamy (unequal sized gametes, with smaller sperm produced by males and larger, flagellated eggs produced by females), and oogamy (unequal sized gametes, unflagellated eggs are much larger than sperm). I, Fertilization external to a multicellular organism versus internal fertilization. $J$, Female colonies versus extrafertile female colonies, which have at least a twofold increase in reproductive cell number. $K$, Male colonies with sperm packets versus dwarf male colonies with sperm packets. Cartoons in $I-K$ are shown with Volvox-like morphology for illustrative purposes only. 
complexity (Parker et al. 1972), and (3) anisogamous species produce larger zygotes than isogamous species (Bulmer and Parker 2002).

\section{Material and Methods}

Phylogeny Estimation

We estimated a new phylogeny for the volvocine green algae (fig. 2; this tree is further analyzed in Hanschen et al. 2018b) that includes 19 strains and species not represented in previous analyses. The in-group was defined as the smallest clade containing Chlamydomonas reinhardtii and Volvox carteri. The out-group consisted of seven nonvolvocine algae, including two taxa from the immediate sister group, one taxon from each of four other major Chlorophyceae groups, and one taxon from the Trebouxiophyceae (out-group taxa were selected on the basis of the phylogeny from Herron and Michod 2008). The sequence data consisted of five chloroplast genes (atpB [ATP synthase beta-subunit], psaA [P700 chlorophyll $a$-apoprotein A1], psaB [P700 chlorophyll $a$-apoprotein A2], $p s b C$ [photosystem II CP43 apoprotein], and $r b c L$ [large subunit of rubisco]; data are deposited in the Dryad Digital Repository: http://dx.doi.org/10.5061/dryad.rr3667m [Hanschen et al. 2018a]). The best partitioning scheme and nucleotide substitution models were determined using PartitionFinder (ver. 2.1.1; Lanfear et al. 2016) with the Akaike information criterion corrected for small sample size (AICc) and a greedy search algorithm with branch lengths linked. Eleven data blocks were determined (three codon positions for five protein-coding chloroplast genes; table A2; tables A1A5 are available online as well as in Dryad: http://dx.doi.org /10.5061/dryad.rr3667m [Hanschen et al. 2018a]).

We generated a concatenated phylogeny (fig. 2) using Bayesian Markov chain Monte Carlo (MCMC) implemented in MrBayes (ver. 3.2.2; Ronquist et al. 2012). Default parameters were used except as described below. Four independent Bayesian analyses of four chains each (three heated chains and one cold chain) were run for $2 \times 10^{7}$ generations with a burn-in of $5 \times 10^{6}$ generations. Trees were sampled every 100 generations. We considered each run to have adequately sampled the solution space when the standard deviation of split frequencies was below $5 \times 10^{-3}$. Post-burn-in trees were combined and assembled to construct a majority-rule consensus phylogram. Posterior probabilities for nodes were calculated using the pooled set of all post-burn-in trees. An ultrametric tree was necessary for maximum likelihood ancestral state reconstruction using the $\mathrm{R}$ package diversitree (FitzJohn 2012). The Bayesian tree was ultrametricized using a penalized likelihood function in the $\mathrm{R}$ package ape (Paradis et al. 2004). A correlated model was used without age constraints. Branch lengths were qualitatively similar to those estimated previously (Herron et al. 2009).
Sequence data for all five chloroplast genes were not available for all taxa (16.5\% of cells in the data matrix were missing). Previous analyses suggest that this low level of missing data should be inconsequential (Wiens and Morrill 2011). Nevertheless, we estimated an additional Bayesian concatenated tree including only taxa for which sequence data for all five chloroplast genes were available (72 of the 97 in-group terminal taxa remained; available in Dryad: http://dx.doi.org/10.5061/dryad.rr3667m [Hanschen et al. 2018a]). PartitionFinder was independently run on this data set, resulting in the same best-fitting partition scheme (table A2). This tree had no strongly supported topological differences from the tree generated from the full data set.

\section{Identification of Character States}

Trait data for each species and strain were compiled from published reports (available in Dryad: http://dx.doi.org/10 $.5061 /$ dryad.rr3667m [Hanschen et al. 2018a]). We gathered data on five discrete sexual traits: (1) isogamy/anisogamy/ oogamy, (2) number of meiotic products hatching (gone cells), (3) external/internal fertilization, (4) presence of dwarf males, and (5) presence of extrafertile females. We gathered data on five continuous metrics: (1) maximum observed number of rounds of cell division $\left(\log _{2}\right.$ (cell number)), (2) maximum observed percentage of somatic cells, (3) maximum observed body length, (4) maximum observed zygote diameter, and (5) anisogamy ratio. The maximum observed and reported values of these metrics were used because many volvocine species can plastically vary the number of rounds of cell division, thus also affecting body size, depending on environmental conditions (Coleman 2012). We calculated the anisogamy ratio (a measure of gamete dimorphism) as the macrogamete volume divided by the microgamete volume, following Randerson and Hurst (2001). This ratio assumes that investment in a sperm packet and an egg is approximately equal.

Following previous authors (Santelices 1999; Clarke 2013; Hanschen et al. 2017), we do not analyze unicellular versus multicellular as a binary trait. Instead, we use three continuous metrics of multicellular complexity: (1) rounds of cell division (i.e., $\log _{2}$ (cell number)), (2) percentage of somatic cells, and (3) natural logarithm of body length. These metrics capture the variation in morphological complexity that is important for multicellularity in the volvocine algae, through traits that reflect increased cell number, division of labor, and secretion of extracellular matrix (a major component of increased body size).

To avoid possible incorrect assignment of character state data due to erroneous species identification or intraspecific variation, care was taken to ensure that data were strain specific rather than species or genus specific. That is, character data were assigned to individual strains on the basis of 


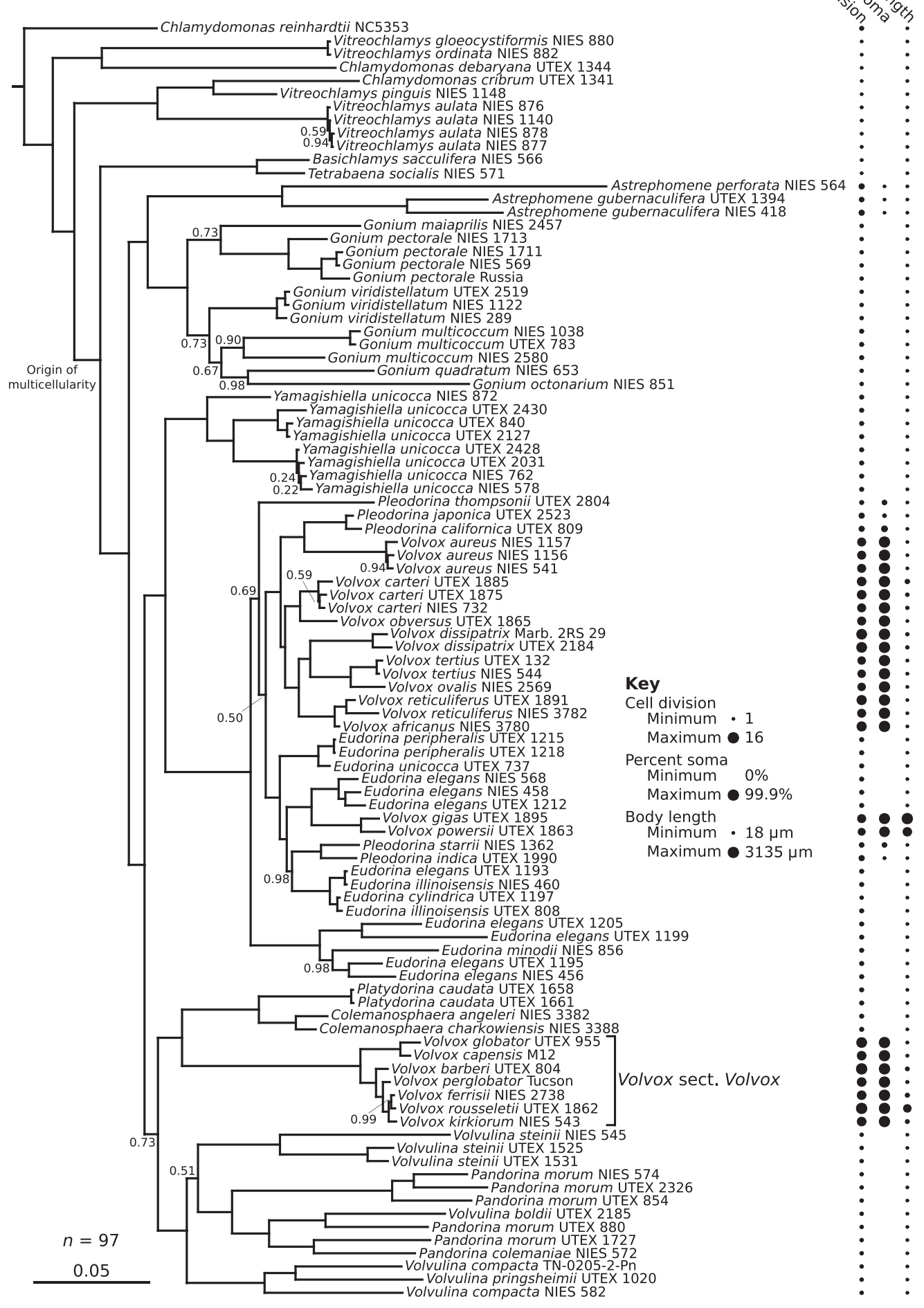

Figure 2: Phylogenetic tree of the volvocine green algae. Numbers indicate Bayesian posterior probabilities (PP). Unlabeled nodes are supported with $\mathrm{PP}=1.00$. Out-group taxa have been trimmed. Circles show relative metrics of multicellular complexity (number of rounds of cell division, percentage of somatic cells, and body length). A key showing minimum and maximum values of metrics is included. The scale bar (for branch lengths) shows expected substitutions per site. 
published data for each strain rather than generalizations made about an entire species or genus.

\section{Measuring Phylogenetic Signal}

We first tested the level of phylogenetic signal in each trait to assess its lability across the tree. For continuous traits, Blomberg's $K$ (Blomberg et al. 2003) and Pagel's $\lambda$ (Pagel 1999) were measured using the R package phytools (Revell 2012; R Core Team 2013). For discrete traits, the $D$ value (Fritz and Purvis 2010) was measured using the R package caper (Orme et al. 2012). All traits had statistically significant phylogenetic signal (tables A3, A4).

\section{Ancestral State Reconstruction}

Ancestral states were reconstructed using maximum likelihood and Bayesian methods. For the maximum likelihood analysis (conducted using diversitree; FitzJohn 2012), several models of character evolution were evaluated for each ancestral state reconstruction, including equal rates of change between states (ER), symmetric rates of gain and loss between states (SYM; only relevant when three states were considered), and all rates different between states (ARD). Models were compared using the AICc (Akaike 1974; Burnham and Anderson 2002). The AICc should identify the best-fitting model without including unnecessary parameters (table A3). When no model of character evolution was strongly preferred $(\Delta \mathrm{AICc}<2)$, reconstructions were performed using both models; in these cases, all reconstructions were robust to the model of character evolution used. The root prior was weighted by the observed frequency of each state among terminal taxa (FitzJohn et al. 2009). In all cases, using alternative root priors (flat prior, two or three given priors) did not affect reconstructions.

The state with the highest probability was considered the most likely for a given node. However, a state was considered to be significantly supported at a given node only if it was at least 7.39 times (if the natural logarithm of the ratio of two likelihoods is greater than 2) more likely than the alternative state (Pagel 1999).

Statistical support for estimated character states at internal nodes was further evaluated using Bayesian hypothesis testing implemented in BayesTraits (ver. 2; Pagel et al. 2004). Phylogenetic uncertainty was explicitly taken into account by analyzing a sample of trees. Every 1,000th post-burn-in tree from the four runs was included, for a total of 600 trees. Outgroups and taxa with unknown character data were trimmed from these trees (e.g., the number of meiotic products hatching has never been observed in Basichlamys sacculifera). A new ultrametric tree was calculated for each trimmed tree using a penalized likelihood function with a correlated model without age constraints (using the $\mathrm{R}$ package ape; Paradis et al.
2004). As above, three models of character evolution (ER, SYM when applicable, and ARD) were analyzed. A Bayes factor (BF) was estimated as twice the difference between the highest harmonic mean log likelihood for each model, calculated from nine independent MCMC runs for each model with 7,500,000 generations (with a burn-in period of 500,000 generations; table A3). The most well-supported model of evolution was used for hypothesis tests for specific ancestral nodes of interest. When no model of character evolution was strongly preferred $(\mathrm{BF}<2)$, nodes of interest were also tested under the alternative model. For specific nodes of interest, the ancestral character state was tested by estimating a BF from five independent MCMC runs with $5,500,000$ generations (with a burn-in period of 500,000 generations) in which the node in question was constrained to one state or the other. Uniform priors and gamma-distributed hyperpriors seeded from a uniform distribution were used to seed all rate parameters. The root state was not set but rather inferred by each MCMC run.

\section{Phylogenetic Statistical Tests}

To test the positive correlation between (binary) sexual characters and the size and complexity of asexual colonies (Parker et al. 1972), we used phylogenetic $t$-tests, implemented in the R package phytools (ver. 0.5-64; Revell 2012). For all phylogenetic $t$-tests regarding multicellular complexity, three covarying metrics were used (natural logarithm of body length, percentage of cells that are somatic, and maximum rounds of cell divisions; fig. 2). Pagel's test (Pagel 1994) was implemented in the R package phytools (Revell 2012) to test for positive correlations between discrete traits. The likelihood ratio test was used to compare alternative maximum likelihood models (i.e., when testing for evolutionary irreversibility of anisogamy; Parker 1982). Identifying which states are derived (fig. 3) allows us to test a positive correlation between the number of derived sexual traits and three metrics of multicellular complexity (fig. 4). In these analyses, we used phylogenetic generalized least squares (PGLS) to regress the number of derived sexual traits (estimated on the basis of the root state in ancestral state reconstructions; fig. 3 ) in each terminal taxon with our three metrics of multicellular complexity. PGLS was implemented using the R package caper (Orme et al. 2012). Analyses included the 65 terminal taxa with available data for all six characters: (1) reduced meiotic products, (2) internal fertilization, (3) anisogamy, (4) oogamy, (5) extrafertile females, and (6) dwarf males. We repeated this analysis including sperm packets as a seventh trait (because sperm are always found in sperm packets, the evolutionary history of sperm packets is identical to anisogamy; fig. $2 B$ ), which did not affect the statistical results (PGLS, adjusted $P<3.69 \times 10^{-7}, r^{2}>0.33$ ). 

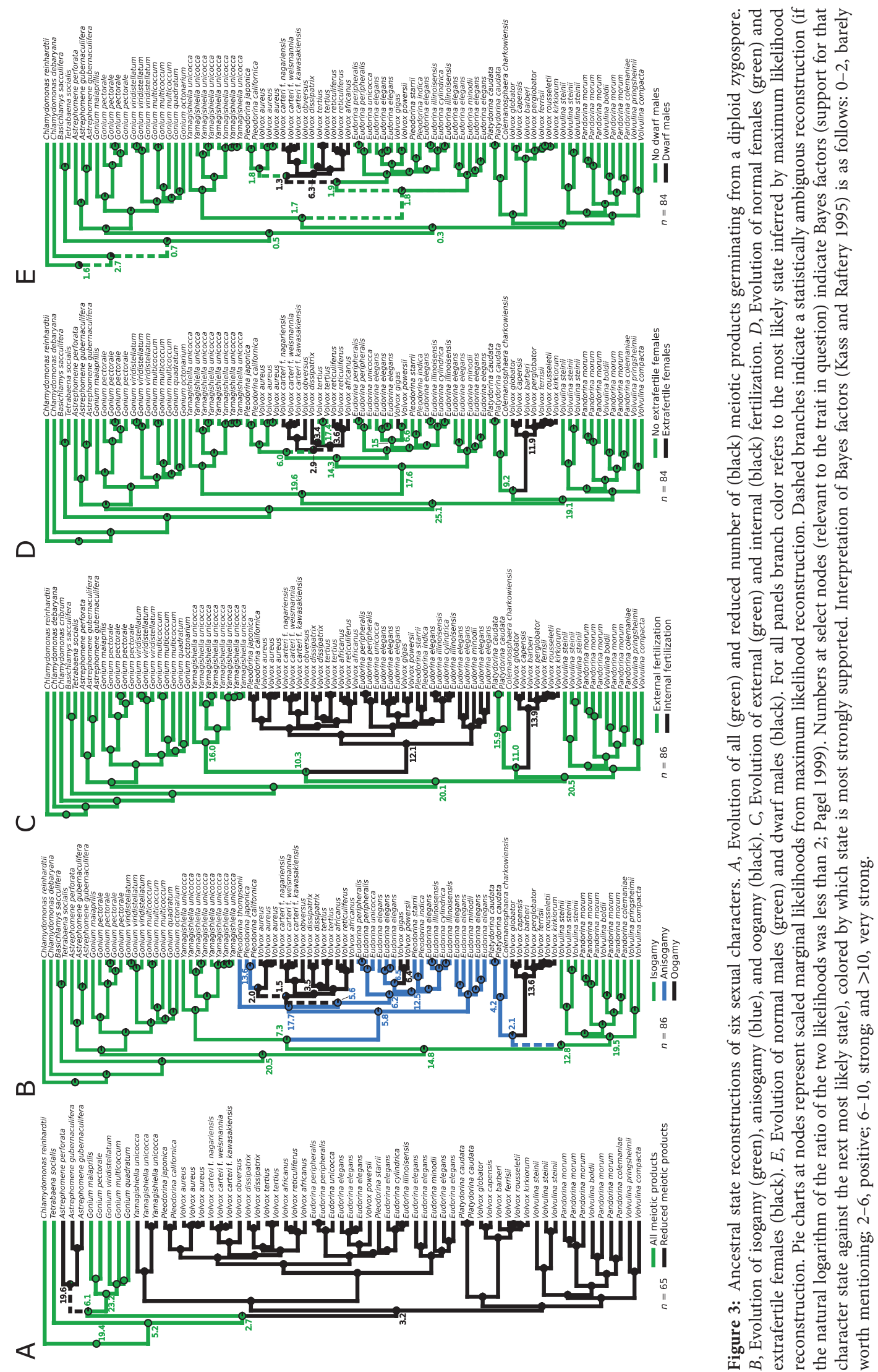

E99 
A

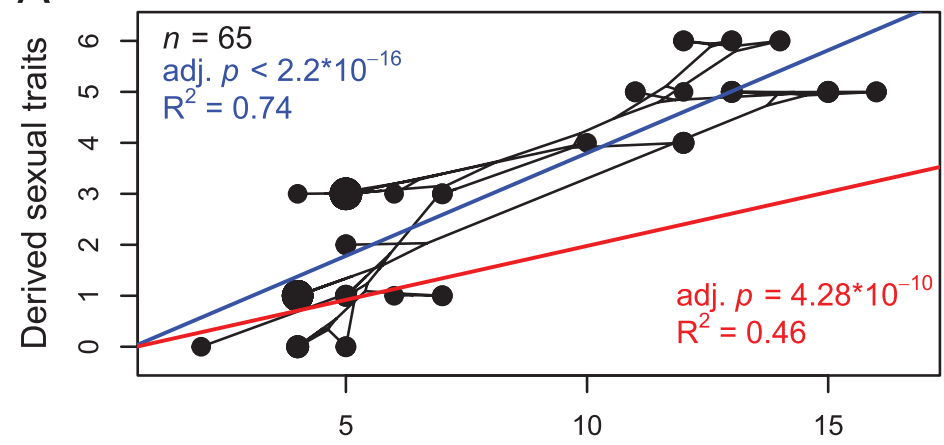

Rounds of cell division

B

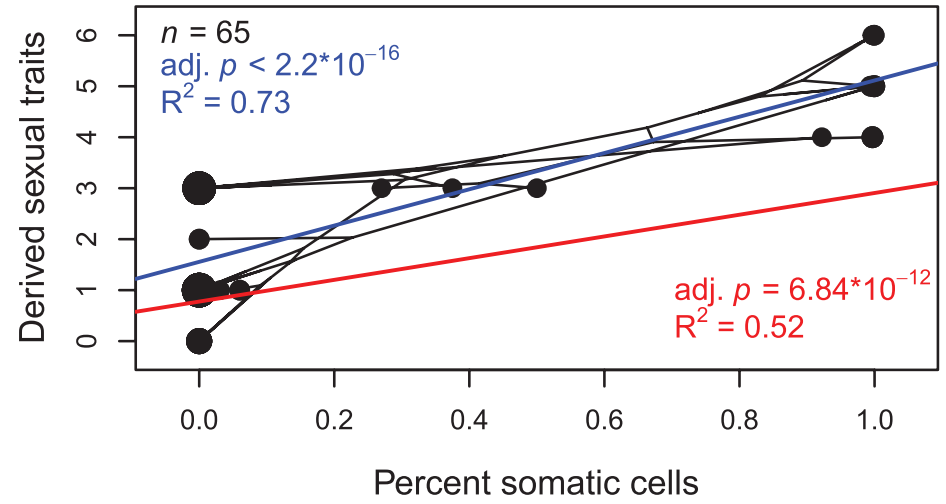

C

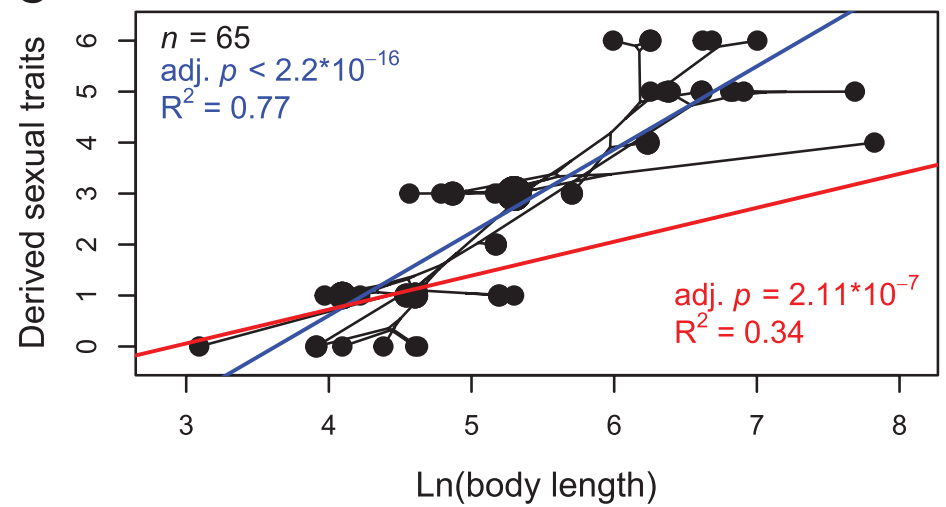

Figure 4: Relationships between the number of derived sexual traits in each species (not including multicellular sperm packets, which are perfectly correlated with anisogamy) and three traits related to multicellular complexity: the number of rounds of cell division in that species $(A)$, the percentage of somatic cells $(B)$, and natural logarithm-transformed body length. Relative sizes of the data points (tips of the phylogeny) are scaled to indicate the number of species at that coordinate (from 1 to 15). Phylogenetic regression lines and statistics (red) and simple linear regressions and statistics (blue) are shown.

\section{Multiple Comparison Adjustments}

When multiple $P$ values were inferred in testing whether a sexual trait correlated with three metrics of multicellularity, $P$ values were adjusted for multiple comparisons following Benjamini and Hochberg (1995).

\section{Results}

\section{Phylogenetic Tree}

The inferred phylogenetic tree of the volvocine green algae is shown in figure 2 (TreeBase ID 21508 and Dryad: http:// dx.doi.org/10.5061/dryad.rr3667m [Hanschen et al. 2018a]). 
This tree included the novel taxa Gonium pectorale Russia and rediscovered Volvox perglobator Tucson. Volvox perglobator Tucson was nested within Volvox sect. Volvox (aka Euvolvox), whereas G. pectorale Russia was nested within G. pectorale (fig. 2). The resulting tree (fig. 2) was concordant with recently published phylogenetic analyses (Nozaki et al. 2006, 2014; Herron and Michod 2008). Any nodes in conflict between our topology and these topologies had low support in our study and in these previous analyses.

\section{Evolution of Volvocine Sexual Traits}

Our ancestral state reconstructions inferred a complex evolutionary history for all sexual traits, including multiple gains and losses (fig. 3). On the basis of ancestral state reconstructions (fig. 3), the first sexual trait to evolve was a reduction in the number of meiotic products hatching from a desiccation-resistant diploid spore (fig. $1 G$ ). This reduction in surviving meiotic products, along with the evolution of polar bodies (the remaining meiotic products), evolved twice: once in the ancestor of the genus Astrephomene and again in the family Volvocaceae (fig. 3A). No relationship between a reduced number of surviving meiotic products and each of three metrics of multicellular complexity was supported (phylogenetic $t$-test, adjusted $P=.216$; table 1).

Anisogamy evolved from isogamy twice independently in the volvocine algae (figs. $1 H, 3 B$ ), providing support for the claim that anisogamous species are "almost certainly derived from isogamous ancestors" (Lehtonen et al. 2016a, p. 1). Anisogamy evolved both times in dioecious, outcrossing ancestors, as opposed to self-fertilizing ancestors. The evolution of oogamy occurred at least three times, likely with one reversion to nonoogamous anisogamy (fig. $3 B$ ). There were no inferred reversions from anisogamy to isogamy (fig. $3 B$ ). To test for evolutionary reversal of anisogamy to isogamy, two models were compared, one constraining the rate of loss of anisogamy to zero and the other constraining the rate of loss to be equal to the rate of gain. Using the likelihood ratio test, the model preventing reversals in anisogamy is not significantly supported $\left(\chi^{2}=1.65, P=.199\right)$.

Consistent with previous theory (Bulmer and Parker 2002), anisogamy (and all other sexual traits) evolved in a multicellular ancestor. Comparing our reconstructions (fig. $3 B$ ) to reconstructions of 12 asexual morphological traits (Kirk 2005; Herron and Michod 2008), 7 of these 12 traits were already present in the ancestors that evolved anisogamy. The ancestor in which anisogamy evolved thus likely resembled extant 16-celled Pandorina or 16-celled Volvulina (figs. 1C, 2, 3).

Table 1: Phylogenetic $t$-test for sexual traits, using three continuous metrics of multicellular complexity (rounds of cell division, percentage of somatic cells, and natural logarithm-transformed body length)

\begin{tabular}{|c|c|c|c|}
\hline Phylogenetic $t$-test (sample size), continuous variable & $t$ & $P$ & Adjusted $P$ \\
\hline \multicolumn{4}{|l|}{ All versus reduced meiotic products $(n=65)$ : } \\
\hline Rounds of cell division & 6.01 & .234 & .306 \\
\hline$\%$ of somatic cells & 4.51 & .306 & .306 \\
\hline $\ln$ (body length) & 13.31 & .072 & .216 \\
\hline \multicolumn{4}{|l|}{ Isogamy versus anisogamy $(n=86)$ : } \\
\hline Rounds of cell division & 50.85 & .045 & .048 \\
\hline$\%$ of somatic cells & 49.32 & .048 & .048 \\
\hline $\ln$ (body length) & 99.74 & .006 & .018 \\
\hline \multicolumn{4}{|l|}{ Isogamy versus anisogamy $(n=72)$ : } \\
\hline $\ln$ (zygote diameter) & 88.81 & .002 & $\ldots$ \\
\hline \multicolumn{4}{|l|}{ External versus internal fertilization $(n=86)$ : } \\
\hline Rounds of cell division & 65.90 & .029 & .033 \\
\hline$\%$ of somatic cells & 63.67 & .033 & .033 \\
\hline $\ln$ (body length) & 117.30 & .009 & .033 \\
\hline \multicolumn{4}{|l|}{ Females versus extrafertile females $(n=84)$ : } \\
\hline Rounds of cell division & 235.14 & .001 & .001 \\
\hline$\%$ of somatic cells & 161.66 & .001 & .001 \\
\hline $\ln$ (body length) & 86.61 & .001 & .001 \\
\hline \multicolumn{4}{|l|}{ Males versus dwarf males $(n=84)$ : } \\
\hline Rounds of cell division & 24.11 & .006 & .009 \\
\hline$\%$ of somatic cells & 33.63 & .001 & .003 \\
\hline $\ln$ (body length) & 14.93 & .026 & .026 \\
\hline
\end{tabular}

Note: A phylogenetic $t$-test comparing natural logarithm-transformed zygote diameter of isogamous and anisogamous algae is included. When multiple metrics were used, $P$ values were adjusted for multiple comparisons following Benjamini and Hochberg (1995). 
Specifically, this ancestor most likely had a colony wall, genetic control of cell number, an organismal anterior-posterior axis, and inversion during development. Therefore, anisogamy evolved in multicellular volvocine ancestors.

Consistent with previous theory (Parker et al. 1972; Bulmer and Parker 2002) and analyses (Knowlton 1974; Bell 1985; Randerson and Hurst 2001), anisogamous algae were significantly larger than isogamous algae (without treating oogamy as a separate character, phylogenetic $t$-test, adjusted $P<.048$; table 1). Additionally, zygotes of anisogamous species were significantly larger than zygotes of isogamous species (phylogenetic $t$-test, $P=.002$; table 1 ). Last, the anisogamy ratio (a measure of gamete dimorphism) significantly increases with multicellular complexity (PGLS, adjusted $P<3.3 \times 10^{-5}$; fig. A2).

Internal fertilization, which is substantially more complex than external fertilization, evolved twice (fig. 3C), broadly corresponding to the evolution of anisogamy (although there are anisogamous species with external fertilization; Nozaki et al. 2014). Internal fertilization was significantly positively correlated with all three metrics of multicellular complexity (phylogenetic $t$-test, adjusted $P<.034$; table 1 ). Internal fertilization was also significantly positively correlated with both anisogamy (Pagel's test, adjusted $P=.026$ ) and oogamy (Pagel's test, adjusted $P=.026$; table A5), which may be expected given that internal fertilization maintains gamete fusion with the evolution of large, less motile gametes (anisogamy and oogamy).

Secondary sexual dimorphism in the form of extrafertile females (fig. 1J), which can increase sexual reproductive output up to 15 times that of asexual reproduction (Coleman 2012), evolved three times independently (fig. 3D) and was lost once (Volvox tertius). Dwarf males (fig. $1 K$ ) evolved once and were subsequently lost in Volvox dissipatrix and Volvox obversus (fig. $3 E$ ). The presence of extrafertile females and dwarf males (both of which occur only in the genus Volvox; Nozaki 2003) were each significantly positively correlated with all three metrics of multicellularity (phylogenetic $t$-test, adjusted $P<.026$; table 1 ). The complexity present in Volvox may be necessary for anisogamy to drive the secondary evolution of sexual dimorphism, as sexual dimorphism is found only in Volvox (Pagel's test of correlation between a species belonging to genus Volvox and the presence of sexual dimorphism, $P=6.04 \times 10^{-5}$ ), even though there are many other anisogamous lineages (fig. $3 B$ ).

The losses of sexual dimorphism occurred along the same branches as independent origins of self-fertilization in $V$. dissipatrix and $V$. tertius (Hanschen et al. 2018b), potentially explaining the loss of sexual dimorphism. However, the rate of loss of sexual dimorphism is not significantly dependent on the rate of the origin of self-fertilization (Pagel's test, likelihood ratio $=1.77, P=.183$ ), likely due to the independent loss of sexual dimorphism (dwarf males) in outcrossing V. obversus (fig. $3 E$ ).

\section{Multicellularity and Sexual Traits}

Species with greater multicellular complexity have significantly more derived sexual characters (PGLS, adjusted $P<2.11 \times 10^{-7}, r^{2}>0.34$; fig. 4). Combined with the observation that all six sexual traits evolved in a multicellular ancestor (figs. 2, 3), this result suggests that multicellularity drove the evolution of sexual traits. Conversely, the prediction that anisogamy drives the evolution of multicellular complexity (Woodland 2016) was not supported. Anisogamy and cellular differentiation were not significantly positively correlated in the volvocine algae (Pagel's test, likelihood ratio $=7.14, P=.129$ ).

\section{Discussion}

We provide empirical evidence supporting the predictions from previous theory (Parker et al. 1972; Bulmer and Parker 2002) that derived sexual traits evolved in a multicellular ancestor (figs. 2,3 ), that they positively correlate with multicellular complexity (table 1), and that anisogamous species produce larger zygotes than isogamous species (table 1). These results are strongly consistent with previous theoretical predictions that multicellularity drives the evolution of costly anisogamy (Bulmer and Parker 2002) and that anisogamy drives the evolution of secondary sexual dimorphism (Lehtonen et al. 2016b). The hypothesis that anisogamy precedes and drives the evolution of multicellular complexity (Woodland 2016) is contradicted by our observations that (1) anisogamy evolved in a multicellular ancestor and (2) anisogamy and cellular differentiation are not correlated. The evolution of cellular differentiation in the isogamous genus Astrephomene (Brooks 1966; Nozaki 1983; Herron and Michod 2008; Grochau-Wright et al. 2017) also contradicts the predicted association between anisogamy and cellular differentiation. The evolution of multicellularity appears to drive the evolution of sexual traits, but the opposite relationship does not appear in the volvocine algae.

The volvocine green algae are broadly consistent with the anisogamy gateway hypothesis. As predicted, (1) we find that anisogamy is strongly positively correlated with increased body size, increased zygote size, and multicellularity; (2) there were no observed reversions from anisogamy to isogamy, although there was not strong statistical support for the irreversibility of anisogamy (possibly due to the limited number of changes overall); and (3) in the volvocine algae, ecological variation may be the previously evolved constraint that prevents invasion of asexual individuals. Only sexually produced diploid zygospores are resistant to temporary environmental variation, such as heat, cold, and desiccation (fig. A1). Asexual individuals are not able to survive periodic heat, cold, and desiccation, preventing the invasion of obligately asexual individuals. 
As predicted, sexual traits significantly positively correlated with multicellular complexity, with the exception of a reduced number of meiotic products. However, a fourfold reduction in surviving meiotic products likely has important evolutionary consequences through a reduction in both the number of and the genetic variation in offspring. Furthermore, the number of meiotic products hatching may interact with the effect of larger zygotes in anisogamous species (table 1), further increasing provisioning per sexual offspring (fig. $3 A, 3 B$ ). The multiple reductions in the number of meiotic products hatching, along with the origins of polar bodies, parallels the independent evolution of polar bodies in plants and animals (Schmerler and Wessel 2011). Using evolutionary repeatability or convergence as an indicator of adaptive value (Simpson 1953; Mayr 1966; Bonner 2000; Schluter 2000), the repeated origins of polar bodies across multicellular taxa suggest that they have an inherent adaptive value, despite their apparent high costs. Therefore, a reduced number of meiotic products may also be caused by multicellularity.

While a reduced number of hatching meiotic products, anisogamy, and internal fertilization were never lost, both forms of sexual dimorphism were lost (fig. 3D, 3E). The loss of sexual dimorphism in Volvox dissipatrix (which lost dwarf males) and Volvox tertius (which lost extrafertile females) may be explained by the evolution of self-fertilization. These losses occurred along the same branches as independent origins of self-fertilization in $V$. dissipatrix and $V$. tertius (Hanschen et al. 2018b). Volvox dissipatrix evolved monoecious selffertilization (i.e., eggs and sperm packets in the same sexual colony; Hanschen et al. 2018b) and therefore does not have male colonies, explaining the loss of dwarf males. Volvox tertius evolved dioecious self-fertilization (i.e., a single genotype produces sperm packets in male colonies and eggs in female colonies; Hanschen et al. 2018b) and has facultative eggs (which do not require sexual differentiation prior to fusion with sperm; Coleman 2012). Other dioecious selfing species (i.e., Volvox aureus) have relatively fewer, facultative eggs, suggesting a connection between dioecious self-fertilization and fewer, facultative eggs. Volvox tertius may have lost extrafertile females due to the origin of dioecious self-fertilization and facultative eggs. Thus, $V$. dissipatrix and $V$. tertius may have similarly lost sexual dimorphism due to similar origins of self-fertilization but lost sexual dimorphism differently by evolving self-fertilization differently. While not statistically significant, the evolution of self-fertilization may nonetheless affect the evolution of sexual complexity.

Ecology might also play an important role in the evolution of anisogamy (Madsen and Waller 1983). Under the fast start hypothesis, species living in stressful environments, such as ephemeral pools, exhibit anisogamy or oogamy because the larger zygospore allows for more rapid growth when necessary (Madsen and Waller 1983). Species living in more stable environments, such as soil, do not require this fast start
(Madsen and Waller 1983). Unfortunately, insufficient information is currently available about volvocine habitats to test this hypothesis. Nevertheless, some observations are consistent with this hypothesis. For example, isogamous Chlamydomonas species occur in soil, and larger-bodied anisogamous/ oogamous genera, such as Pleodorina and Volvox, do live in ephemeral pools and lakes (Kirk 1998; Harris 2009). It is also possible that a soil habitat does not select for the evolution of multicellularity, while an aqueous environment does (perhaps because of the presence of invertebrate filter feeders; Bell 1985; Kirk 1998). If so, this would lead to an apparent positive correlation between multicellularity and anisogamy when both are instead driven by habitat. However, the presence of anisogamous, unicellular algae (Oogamochlamys and Lobochlamys; Ettl 1983; Pröschold et al. 2001) and isogamous, multicellular algae (such as Pandorina and Volvulina; figs. 2, 3) serve as two counterexamples to the hypothesis that habitat drives the joint evolution of anisogamy and multicellularity. Of course, the hypotheses discussed here are not mutually exclusive; habitat may drive the evolution of multicellularity while both habitat and multicellularity drive the evolution of anisogamy.

Conclusions. We demonstrate the evolutionary relationship between the diverse forms of multicellular complexity and the diverse forms of sexual reproduction in the volvocine green algae. These sexual traits are shared by other multicellular taxa, highlighting the evolutionary convergence among multicellular taxa and suggesting that the evolution of traits related to multicellularity and sexuality in the volvocine algae may offer a window into their origins and evolution across the Tree of Life.

\section{Acknowledgments}

We thank Dinah R. Davison, Patrick J. Ferris, and Daniel S. Moen for technical assistance; Deborah E. Shelton for collecting strains; and Dinah R. Davison, Patrick J. Ferris, and Sarah P. Otto for their helpful comments and discussion. We acknowledge the support of the National Aeronautics and Space Administration (NNX13AH41G, NNX15AR33G, cooperative agreement notice 7), the National Institutes of Health (GM084905), the National Science Foundation (MCB-1412395, DEB-1457701), and Ministry of Education, Culture, Sports, Science, and Technology (MEXT)/Japan Society for the Promotion of Science (JSPS) Grants-in-Aid for Scientific Research (KAKENHI 15K14590, 16H02518).

Statement of authorship: E.R.H. conceived the project, collected species character data, described strains, sequenced chloroplast genes, constructed the species tree, performed statistical analyses, and wrote the manuscript; M.D.H. conceived the project and collected species character data; J.J.W. conceived the experimental design; H.D.N. collected species 
character data; R.E.M. conceived the project; and all authors contributed to preparation of the manuscript.

\section{Literature Cited}

Akaike, H. 1974. A new look at the statistical model identification. IEEE Transactions on Automatic Control 19:716-723.

Bateman, A. J. 1948. Intra-sexual selection in Drosophila. Heredity 2:349-368.

Bell, G. 1978. The evolution of anisogamy. Journal of Theoretical Biology 73:247-270.

. 1982. The masterpiece of nature: the evolution and genetics of sexuality. Croom Helm, London.

. 1985. The origin and evolution of germ cells as illustrated by the Volvocales. Pages 221-256 in H. O. Halvorson and A. Monroy, eds. The origin and evolution of sex. Alan R. Liss, New York.

Benjamini, Y., and Y. Hochberg. 1995. Controlling the false discovery rate: a practical and powerful approach to multiple testing. Journal of the Royal Statistical Society B 57:289-300.

Beukeboom, L., and N. Perrin. 2014. The evolution of sex determination. Oxford University Press, Oxford.

Blomberg, S. P., T. Garland, and A. R. Ives. 2003. Testing for phylogenetic signal in comparative data: behavioral traits are more labile. Evolution 57:717-745.

Bonner, J. T. 2000. First signals: the evolution of multicellular development. Princeton University Press, Princeton, NJ.

Brooks, A. 1966. The sexual cycle and intercrossing in the genus Astrephomene. Journal of Eukaryotic Microbiology 13:367-375.

Bulmer, M. G., and G. A. Parker. 2002. The evolution of anisogamy: a game-theoretic approach. Proceedings of the Roval Societv B 269:2381-2388.

Burnham, K. P., and D. R. Anderson. 2002. Model selection and multimodel inference: a practical information-theoretic approach. Springer, New York.

Clarke, E. 2013. The multiple realizability of biological individuals. Journal of Philosophy 110:413-435.

Coleman, A. W. 2012. A comparative analysis of the Volvocaceae (Chlorophyta). Journal of Phycology 48:491-513.

Ettl, H. 1983. Chlorophyta I: Phytomonidia. H. Ettl, J. Gerloff, H. Heynig, and D. Mollenhauer, eds. Süsswasserflora von Mitteleuropa. Gustav Fischer, Stuttgart.

Featherston, J., Y. Arakaki, E. R. Hanschen, P. J. Ferris, R. E. Michod, B. J. S. C. Olson, H. Nozaki, and P. M. Durand. 2018. The 4-celled Tetrabaena socialis nuclear genome reveals the essential components for genetic control of cell number at the origin of multicellularity in the volvocine lineage. Molecular Biology and Evolution 35:855-870.

FitzJohn, R. G. 2012. Diversitree: comparative phylogenetic analyses of diversification in R. Methods in Ecology and Evolution 3:10841092.

FitzJohn, R. G., W. P. Maddison, and S. P. Otto. 2009. Estimating traitdependent speciation and extinction rates from incompletely resolved phylogenies. Svstematic Biology 58:595-611.

Fritz, S. A., and A. Purvis. 2010. Selectivity in mammalian extinction risk and threat types: a new measure of phylogenetic signal strength in binary traits. Conservation Biology 24:1042-1051.

Grochau-Wright, Z. I., E. R. Hanschen, P. J. Ferris, T. Hamaji, H. Nozaki, B. J. S. C. Olson, and R. E. Michod. 2017. Genetic basis for soma is present in undifferentiated volvocine green algae. Lournal of Evolutionary Biology 30:1205-1218.
Hanschen, E. R., D. R. Davison, Z. I. Grochau-Wright, and R. E. Michod 2017. Evolution of individuality: a case study in the volvocine green algae. Philosophy, Theory, and Practice in Biology 9:3.

Hanschen, E. R., M. D. Herron, J. J. Wiens, H. Nozaki, and R. E. Michod. 2018a. Data from: Multicellularity drives the evolution of sexual traits. American Naturalist, Dryad Digital Repository, http:dx.doi.org /10.5061/dryad.rr3667m.

2018b. Repeated evolution and reversibility of self-fertilization in the volvocine green algae. Evolution 72:386-398.

Hanschen, E. R., T. N. Marriage, P. J. Ferris, T. Hamaji, A. Toyoda, A. Fujiyama, R. Neme, et al. 2016. The Gonium pectorale genome demonstrates co-option of cell cycle regulation during the evolution of multicellularity. Nature Communications 7:11370.

Harris, E. H. 2009. The Chlamydomonas sourcebook. Vol. 1. 2nd ed. Academic Press, San Diego, CA.

Herron, M. D., J. D. Hackett, F. O. Aylward, and R. E. Michod. 2009. Triassic origin and early radiation of multicellular volvocine algae. Proceedings of the National Academv of Sciences of the USA 106: 3254-3258

Herron, M. D., and R. E. Michod. 2008. Evolution of complexity in the volvocine algae: transitions in individuality through Darwin's eye. Evolution 62:436-451.

Kass, R. E., and A. E. Raftery. 1995. Bayes factors. Journal of the American Statistical Association 90:773-795.

Kirk, D. L. 1998. Volvox: molecular-genetic origins of multicellularity and cellular differentiation. Cambridge University Press, Cambridge.

2005. A twelve-step program for evolving multicellularity and a division of labor. BioEssavs 27:299-310.

Kirk, D. L., and M. M. Kirk. 1986. Heat shock elicits production of sexual inducer in Volvox. Science 231:51-54.

Knowlton, N. 1974. A note on the evolution of gamete dimorphism. Journal of Theoretical Biology 45:283-285.

Lanfear, R., P. B. Frandsen, A. M. Wright, T. Senfeld, and B. Calcott. 2016. PartitionFinder 2: new methods for selecting partitioned models of evolution for molecular and morphological phylogenetic analyses. Molecular Biology and Evolution 34:772-773.

Lehtonen, J., H. Kokko, and G. A. Parker. 2016a. What do isogamous organisms teach us about sex and the two sexes? Philosophical Transactions of the Roval Society B 371:20150532.

Lehtonen, J., G. A. Parker, and L. Schärer. 2016b. Why anisogamy drives ancestral sex roles. Evolution 70:1129-1135.

Madsen, J. D., and D. M. Waller. 1983. A note on the evolution of gamete dimorphism in algae. American Naturalist 121:443-447.

Maynard Smith, J. 1978. The evolution of sex. Cambridge University Press, Cambridge.

. 1982. Evolution and the theory of games. Cambridge University Press, Cambridge.

Mayr, E. 1966. Animal species and evolution. Harvard University Press, Cambridge, MA.

Merchant, S. S., S. E. Prochnik, O. Vallon, E. H. Harris, J. Karpowicz, G. B. Witman, A. Terry, et al. 2007. The Chlamydomonas genome reveals the evolution of key animal and plant functions. Science 318:245-250.

Nozaki, H. 1983. Morphology and taxonomy of two species of Astrephomene (Chlorophyta) in Japan. Journal of Japanese Botany 58:345-352.

2003. Flagellated green algae. Pages $225-252$ in J. D. Wehr and R. G. Sheath, eds. Freshwater algae of North America. Academic Press, San Diego, CA.

Nozaki, H., and M. Itoh. 1994. Phylogenetic relationships within the colonial Volvocales (Chlorophyta) inferred from cladistic analysis based on morphological data. Journal of Phycology 30:353-365. 
Nozaki, H., F. D. Ott, and A. W. Coleman. 2006. Morphology, molecular phylogeny and taxonomy of two new species of Pleodorina (Volvoceae, Chlorophyceae). Journal of Phycology 42:1072-1080.

Nozaki, H., T. K. Yamada, F. Takahashi, R. Matsuzaki, and T. Nakada. 2014. New "missing link" genus of the colonial volvocine green algae gives insights into the evolution of oogamy. BMC Evolutionary Biology 14:37.

Orme, C. D. L., R. P. Freckleton, G. H. Thomas, T. Petzoldt, S. A. Fritz, N. J. B. Isaac, and W. Pearse. 2012. The caper package: comparative analysis of phylogenetics and evolution in R. R package version 0.5 .

Pagel, M. 1994. Detecting correlated evolution on phylogenies: a general method for the comparative analysis of discrete characters. Proceedings of the Roval Societv B 255:37-45.

. 1999. The maximum likelihood approach to reconstructing ancestral character states of discrete characters on phylogenies. Systematic Biology 48:612-622.

Pagel, M., A. Meade, and D. Barker. 2004. Bayesian estimation of ancestral character states on phylogenies. Systematic Biology 53:673-684.

Paradis, E., J. Claude, and K. Strimmer. 2004. APE: analyses of phylogenetics and evolution in R language. Bioinformatics 20:289-290.

Parker, G. A. 1982. Why are there so many tiny sperm? sperm competition and the maintenance of two sexes. Iournal of Theoretical Biology 96:281-294.

Parker, G. A., R. R. Baker, and V. G. F. Smith. 1972. The origin and evolution of gamete dimorphism and the male-female phenomenon. Journal of Theoretical Biology 36:529-553.

Prochnik, S. E., J. Umen, A. M. Nedelcu, A. Hallmann, S. M. Miller, I. Nishii, P. J. Ferris, et al. 2010. Genomic analysis of organismal complexity in the multicellular green alga Volvox carteri. Science 329:223-226.

Pröschold, T., B. Marin, U. G. Schlösser, and M. Melkonian. 2001. Molecular phylogency and taxonomic revision of Chlamydomonas (Chlorophyta). I. Emendation of Chlamydomonas ehrenberg and Chloromonas gobi, and description of Oogamochlamys gen. nov. and Lobochlamys gen. nov. Protist 152:265-300.

Randerson, J. P., and L. D. Hurst. 2001. A comparative test of a theory for the evolution of anisogamy. Proceedings of the Roval Society B 268:879-884.

R Core Team. 2013. R: a language and environment for statistical computing. R Foundation for Statistical Computing, Vienna.

Revell, L. J. 2012. phytools: an R package for phylogenetic comparative biology (and other things). Methods in Ecology and Evolution 3:217-223.

Ronquist, F., M. Teslenko, P. van der Mark, D. L. Ayres, A. Darling, S. Hohna, B. Larget, L. Liu, M. A. Suchard, and J. P. Huelsenbeck. 2012. MrBayes 3.2: efficient Bayesian phylogenetic inference and model choice across a large model space. Systematic Biology 61: 539-542.

Santelices, B. 1999. How many kinds of individual are there? Trends in Ecology and Evolution 14:152-155.

Schluter, D. 2000. The ecology of adaptive radiation. Oxford University Press, Oxford.

Schmerler, S., and G. Wessel. 2011. Polar bodies - more a lack of understanding than a lack of respect. Molecular Reproduction and Development 78:3-8.

Simpson, G. G. 1953. The major features of evolution. Columbia University Press, New York.

Smith, G. M. 1944. A comparative study of the species of Volvox. Transactions of the American Microscopical Society 63:265-310.

Togashi, T., and P. A. Cox. 2011. The evolution of anisogamy. Cambridge University Press, Cambridge.

Wiens, J. J., and M. C. Morrill. 2011. Missing data in phylogenetic analysis: reconciling results from simulations and empirical data. Svstematic Biology 60:719-731.

Woodland, H. R. 2016. The birth of animal development: multicellularity and the germline. Pages 609-630 in P. M. Wassarman, ed. Current topics in developmental biology. Vol. 117. Academic Press, Burlington, MA.

\section{References Cited Only in the Online Appendixes}

Hamaji, T., P. J. Ferris, I. Nishii, Y. Nishimura, and H. Nozaki. 2013. Distribution of the sex-determining gene MID and molecular correspondence of mating types within the isogamous genus Gonium (Volvocales, Chlorophyta). PLoS ONE 8:e64385.

Isaka, N., H. Kawai-Toyooka, R. Matsuzaki, T. Nakada, and H. Nozaki. 2012. Description of two new monoecious species of Volvox sect. Volvox (Volvocaceae, Chlorophyceae), based on comparative morphology and molecular phylogeny of cultured material. Journal of Phycology 48:759-767.

Janet, C. 1912. Le Volvox. Imprimerie-librairie Ducourtieux et Gout, Limoges.

Miller, S. M., and D. L. Kirk. 1999. glsA, a Volvox gene required for asymmetric division and germ cell specification, encodes a chaperonelike protein. Development 126:649-658.

Nozaki, H., M. Itoh, R. Sano, H. Uchida, M. M. Watanabe, and T. Kuroiwa. 1995. Phylogenetic relationships within the colonial Volvocales (Chlorophyta) inferred from $r b c L$ gene sequence data. Lournal of Phycology 31:970-979.

Associate Editor: David N. Reznick Editor: Alice A. Winn 